\title{
Tooley's Solution to the Inference Problem*
}

TheOdore Sider Pbilosophical Studies 67 (1992): 26I-75

In response to various shortcomings of regularity theories of natural law, some philosophers of a realist bent have recently been drawn to the view that a law of nature is a relation between universals. Heading this group are Michael Tooley and D. M. Armstrong. ${ }^{1}$

The view that laws are relations between universals may have great theoretical promise, but it faces a problem of its own. Surely, if it is a law of nature that $F$ s are $G$ s then it follows that all $F$ s are Gs. But for views like those of Armstrong and Tooley this entailment seems to be a mystery. In this paper I discuss Tooley's attempt in his recent book Causation to solve what has become known as the "inference problem".

\section{The Solution by Stipulation}

Suppose there is a law that all $F \mathrm{~s}$ are $G s .^{3}$ On Tooley's view, this fact consists in the universal $F$ standing in a certain relation, $R$, called the relation of "nomic necessitation", to the universal $G$. But the existence of this law entails the corresponding generalization that all $F$ S are Gs. So (I) must entail (2):
(I) $F$ bears $R$ to $G$
(2) All $F$ s are $G \mathrm{~s}$

But how can this be? Calling $R$ "nomic necessitation" merely indicates that the entailment must exist. But why should some state of affairs in the lofty realm of universals, a fancy relation's holding between two universals, translate into hard facts below, facts about earthly particulars that fall under those universals? Tooley must provide an account of the nature of $R$ that shows why (I) entails (2).

*I would like to thank Phillip Bricker for his persistent help with this paper. I would also like to thank David Lewis and an anonymous referee for Philosophical Studies for their comments.

${ }^{1}$ Tooley (1987); Armstrong (1983).

${ }^{2}$ See Tooley (I987, 76-91, I IO-I I 2, and I 23-I 29). Van Fraassen introduced the phrase "inference problem" in van Fraassen (1989, chapter 5). David Lewis presses the inference problem in Lewis (1986, xii).

${ }^{3}$ In this paper I consider only laws of the simple form all Fs are Gs. 
Bas van Fraassen distinguishes the "inference problem" from the "identification problem". ${ }^{4}$ One question is how we could ever single out $R$ from all of the relations between universals. This is the identification problem. A separate though related problem, the inference problem, is that of how $R$ does its stuff. How could any relation play the role that the relation of nomic necessitation must play in making (I) entail (2)?

Tooley's solution to the identification problem I will call the solution by stipulation. He simply stipulates that the necessitation relation by definition is one that has the properties it needs to deserve the name 'necessitation relation'.

More exactly, Tooley offers a set $S$ of properties that the nomic necessitation relation is intended to have, and then applies the familiar Ramsey-Lewis method for defining theoretical terms. ${ }^{5}$ For example, the relation must be "irreducibly" a relation between universals, for if it were analyzable in terms of facts about particulars then Tooley's view would collapse into a regularity theory. $R$ must also be capable of holding contingently between universals since the laws of nature are, presumably, contingent. Most importantly, $R$ must be such that if $F$ bears it to $G$ then every particular that instantiates $F$ must also instantiate $G$. Let us call any relation that possesses all the properties in $S$ a "Tooley relation". The nomic necessitation relation, then, is defined as the unique Tooley relation. Given any sentence $\phi(\tau)$ that contains occurrences of a term $\tau$ purporting to refer to the relation of nomic necessitation (and no unbound occurrences of the variable $R$ ), we "Ramsify" it, replacing it with an existentially quantified sentence:

There exists a unique Tooley relation, $R$, such that $\phi(R / \tau)$

(where $\phi(R / \tau)$ is the result of replacing all occurrences of $\tau$ in $\phi$ with the variable $R$ ).

The solution by stipulation is, of course, somewhat unsatisfying. It does not tell us what $R$ is like. It merely states that it is a relation with the properties listed in $S$. We may still wonder how there could be a relation with the powers $R$ is supposed to have, such that if $F$ and $G$ stand in it, then all $F$ s must be $G$ s. How does $R$ do its stuff? The identification problem may be solved, but the inference problem remains.

The lack of an account of how $R$ does its stuff is not fatal to Tooley. Perhaps the failure of competing accounts of natural law justifies a faith that there

\footnotetext{
${ }^{4}$ van Fraassen (1989, 38-39).

${ }^{5}$ See Tooley (1987, 78-80).
} 
is some Tooley relation out there. Our partial ignorance of its nature does not prove its nonexistence. But it would be nicer to have an account of how a relation could be thus. To this end, Tooley offers a second theory. His speculative theory attempts to give us an illuminating account of how there could be a Tooley relation.

\section{The Speculative Theory}

The distinctive feature of the speculative theory is its application of the partwhole relation to universals. Again, suppose that in some possible world w there is a law that all $F$ s are Gs. $F$ must bear the relation of nomic necessitation to $G$. This fact, speculates Tooley, is just the fact that the universal $F$ exists, in $w$, "only as a part of" the conjunctive universal $F \& G{ }^{6}$

We will investigate presently the question of whether Tooley's speculative theory can solve the inference problem. But first I want to discuss his application of part/whole concepts to universals.

Tooley's universals are transcendent universals, not mere abstractions from concrete particular states of affairs (as are the immanent universals of someone like Armstrong ${ }^{7}$ ). Tooley's universals do not depend on concrete particulars for their existence; on his view, it is possible that a universal exist with no instances. The intuitive picture is that they are other-worldly "abstract" entities that inhabit a realm entirely separate from that of the material world, to which they are linked by the relation of instantiation. In such a realm, how does the part-whole relation apply?

I do not claim that we cannot apply mereology to transcendent universals. But the application should be conservative, relying only on what is uncontroversial about part/whole relations and universals. Appeals to, say, the transitivity of the parthood relation in the realm of universals would be uncontroversial. But radical principles and controversial inferences should be distrusted. We might call principles like the following:

Every universal is a mereological atom

Every universal has proper parts

Any fusion of universals must be a universal

Fusions of distinct universals are never universals

\footnotetext{
${ }^{6}$ Tooley (I987, I $23 \mathrm{ff}$.)

${ }^{7}$ See Armstrong $(1978 a, b)$.
} 
"radical". They concern how universals interact with part-whole relations, making claims that go beyond what is uncontroversial about parts and wholes and universals. The domain of universals is unfamiliar to part/whole concepts, especially because Tooley's universals are transcendent universals. Therefore, unless defended by convincing arguments, radical principles have no special claims on our intuitions. Appeals to radical principles will be suspect, and if Tooley's speculative theory must make essential use of radical principles, then I think it forfeits its claim to be genuinely illuminating.

These considerations prompt the following warning to Tooley: beware that you do not forfeit your claim to illuminate, lest you make no improvement on the solution by stipulation! We must be clear here about the goal of the speculative theory. The notion of a Tooley relation is initially mysterious. The speculative theory seeks to dispel some of that mystery. To make clear to us how there could be such a thing, the speculative theory must not introduce mysteries greater than the original. Appeal to radical principles would do just this.

The import of my warning will become clearer as I develop my main criticism of Tooley's speculative theory. I will argue that the speculative theory is of no help whatsoever in solving the inference problem. Suppose we have a law that $F$ s are $G$ s. The speculative theory then says:

(3) the universal $F$ exists only as a part of the universal $F \& G$.

But how are we to infer that all $F$ s are $G$ s? If (3) entailed

(4) any particular that bears the instantiation relation to $F$ bears the instantiation relation to $F \& G$

then we would have a solution, for (4) says that all $F$ s are Gs. Tooley seems to think that the inference is so unproblematic as to not even require comment. Here is the only passage on this inference in Causation: ${ }^{8}$

If it is a law that everything with property $P$ has property $Q$, then the universal which is property $P$ exists, in that world, only as a part of the conjunctive universal $P$ and $Q$. Therefore, the only way that property $P$ can be instantiated in a given individual, in a world where that law obtains, is if the conjunctive universal is instantiated in that individual.

\footnotetext{
${ }^{8}$ Tooley $(1987$, I 28-9).
} 
Unfortunately, we cannot evaluate this inference until we know what the phrase ' $F$ exists only as a part of $F \& G$ ' means. The phrase is obscure, and there are few passages that give us interpretational clues. The following quotation shows that it is safe to assume that if $x$ exists only as a part of $y$ then $x$ is a part of $y:^{9}$

...Some realists...reject the idea of complex universals. But if all universals are simple, none is a part of any other, and, a fortiori, there cannot be any that exist only as parts of other universals.

But beyond this rather basic constraint we are left on our own.

There is another interpretational issue. ${ }^{10}$ As we will see, on all plausible interpretations of the phrase 'exists only as a part of', the inference from (3) to (4) is extremely problematic, and yet Tooley writes as if this inference is transparently clear. Why?

One explanation is as follows. As noted, it is clear that Tooley takes ' $U$ exists only as a part of $V$ ' to definitionally entail ' $U$ is a part of $V$ '. So if Tooley were using the phrase ' $U$ is a part of $V$ ' in such a way that it definitionally entails 'all $U$ s are $V s$ ', then (3) would indeed definitionally entail (4), and we would have our explanation of Tooley's lack of comment on the inference.

One way for ' $U$ is a part of $V$ ' to definitionally entail 'all $U$ s are $V$ s' would be if the former phrase meant the same as the latter, by definition. From the text, it appears that Tooley does not accept this definition. ${ }^{11}$ Moreover, this definition has an interesting consequence. Suppose we have a law that $U s$ are $V$ s. The speculative theory says that, at the world in question, $U$ exists only as a part of $U \& V$. It follows that $U$ is a part of $U \& V$. But by the definition under consideration, a conjunctive universal is always a part of each of its conjuncts. Hence, $U$ is also a part of $U \& V$. But this entails that those universals are identical. Surely this is too strong a result, but I defer discussion of this possibility until section 3 .

Surely then, ' $U$ is a part of $V$ ' does not simply mean 'All $U$ s are $V$ s'. But perhaps Tooley has some other definition in mind for 'part' as applied to universals according to which 'all $U$ s are $V$ s' is necessary, but not sufficient,

\footnotetext{
${ }^{9}$ Tooley (1987, I 24).

${ }^{10} \mathrm{I}$ am indebted to Phillip Bricker for suggestions here.

${ }^{11}$ The paragraph in Tooley ( 1987 ) that begins on the bottom of p. I 24 and continues into p. I 25 seems to contain a claim that a Factual Platonic realist (such as Tooley), unlike an Aristotelian realist, need not accept this definition, and that its rejection is crucial to the speculative theory. Unfortunately, this paragraph is somewhat mystifying. Note the shift from discussion of existence only as a part to discussion of parthood.
} 
for ' $U$ is a part of $V$ '. That is, the possibility I am considering is that Tooley accepts some definition of the following form:

(P) universal $U$ is a part of universal $V$ iff all $U$ s are $V$ s and -

where the blank on the right hand side is filled in in some way or another. Tooley does not mention any such principle. Moreover, depending on how the blank is filled in and what the phrase 'exists only as a part of' means, this might violate Tooley's requirement we noted in the first section: that the necessitation relation between universals not be reducible to facts about particulars. Still, Tooley's lack of comment on the inference from (3) to (4) requires explanation; his acceptance of a definition of the form $(\mathrm{P})$ would be such an explanation.

We have, then, two distinct questions of interpretation. The first is the question of the meaning of 'exists only as a part of'. The second is the question of the status of parthood for universals. Does Tooley accept a definition of the form $(\mathrm{P})$, or does he give no particular analysis of the part-whole relation for universals? This generates a plethora of possible theories; the rest of the section is devoted to their investigation. I will argue that all are unacceptable. Each theory will involve a particular interpretation of 'exists only as a part of' and a particular decision as to whether some definition of the form $(\mathrm{P})$ is accepted. I supply the following chart:

\section{Speculative Theories}

\begin{tabular}{lll} 
Theory & ' $x$ exists only as a part of $y$ ' means? & (P) defines 'part'? \\
\hline I & ' $x$ is a part of $y$ ' & no \\
2 & ' $x$ is a part of $y$ ' & yes \\
3 & ' $x$ is a part only of $y$ ' & \\
4 & ' $x$ is a part of $y$ and $x$ is not part of any universal \\
& that is not a part of $y$ ' & \\
5 & ' $x$ is a part of and ontologically depends on $y$ ' & no \\
6 & ' $x$ is a part of and ontologically depends on $y$ ' & yes
\end{tabular}

\section{Theory $I$}

Perhaps ' $F$ exists only as a part of $F \& G$ ' means that $F$ is a part of $F \& G$ and additionally that $F$ "has no existence apart from" $F \& G$; that is, that $F$ has no parts that are wholly disjoint from (i.e. do not overlap) $F \& G$. But this is just a roundabout way of saying that $F$ is a part of $F \& G$. (3), so interpreted, means simply: 


\section{(3a) Universal $F$ is a part of universal $F \& G$}

If this is what Tooley means by (3), then the presence of 'only' in the original is a little puzzling. Still, I find (3a) a natural interpretation of (3); moreover, I will draw on what I say here in my discussions of the other possibilities for the Speculative Theory.

Theory I, then, interprets 'exists only as a part' as meaning simply 'is a part of'. Let us also say that Theory I does not contain any particular definition of the form $(\mathrm{P})$ of the part-whole relation for universals. This theory, I think, does not solve the inference problem. The move from (3a) to (4) here is not straightforward. From the fact that a part of the universal $F \& G$, namely $F$, is instantiated in some particular $a$, why should it follow that (the whole of) $F \& G$ is instantiated in $a$ ? The principle behind this inference seems to be:

(?) For any object $x$ and universals $U$ and $V$, if $U$ is a part of $V$, and $x$ instantiates $U$, then $x$ must instantiate $V$ as well

But Tooley gives us no argument for (?). And (?) is by no means obvious. In fact, it seems prima facie doubtful. If $\boldsymbol{F}$ is a part of $\boldsymbol{F} \& G$, it seems that $F$ might be a proper part of $F \& G$, and that the part of $F \& G$ which is distinct from $F$ may be the "G part", so an object $a$ might fail to be $G$ (and hence fail to be $F \& G$ ) despite its being $F$.

(?) is a paradigm of what I called a "radical principle". It is not intuitively obvious, nor is it, as far as I can tell, a consequence of uncontroversial principles relating either to universals or part/whole relations. For example, Tooley cannot argue for the truth of (?) from any general principle about parts and wholes such as:

(?') For any objects $x, y$, and $z$, and any relation $R$, if $y$ is a part of $z$ and $x$ bears $R$ to $y$, then $x$ bears $R$ to $z$

for (?') is false (I am capable of having a piece of cake in my mouth without having the whole cake in there as well).

We could restrict (?') to the instantiation relation:

(?") For any objects $x, y$, and $z$, if $y$ is a part of $z$ and $x$ instantiates $y$, then $x$ must instantiate $z$ also. 
But (?") is clearly false also. Suppose unit negative charge is a universal. Many electrons instantiate this universal. But consider various mereological sums of the universal unit negative charge with other entities. We could fuse unit negative charge with the Statue of Liberty, or with some other universal such as the universal unit positive charge; in each case unit negative charge is a part of the fusion. But surely no electron instantiates either of these fusions.

In the absence of any justification for the radical principle (?), I think we should conclude that (4) does not follow in any straightforward way from (3a). As I said above, it seems intuitively possible that $F$ be a proper part of $F \& G$, and possible for some particular to instantiate the " $F$-part" of $F \& G$ without instantiating the " $G$-part". ${ }^{2}$

\section{Theory 2}

Here we keep the definition of 'exists only as a part of' from Theory I, but add some definition of the form $(\mathrm{P})$. This interpretation has the virtue of making the relation between ( $3 \mathrm{a}$ ) and (4) be that of transparent entailment. But surely this cannot be Tooley's speculative theory. What could go in the blank $(-)$ in $(\mathrm{P})$ ? Let us draw back and examine the claim of Theory 2 . Removed of the trappings, Theory 2 says that there is a law that $F$ s are $G$ s iff all $F$ s are $G s$ and -. If we knew how to fill in that blank, then the game would be finished, for what goes in the blank is whatever turns a mere regularity into a law! But of course, this is the very question any theory of laws is trying to answer.

\section{Theory 3}

How else can we understand 'exists only as a part of'? On a reading that is somewhat faithful to the surface structure of (3), that sentence means that $F$ is a part of $F \& G$, and only of $F \& G$; that is:

(3b) $F$ is a part of $F \& G$ and $F$ is not a part of anything else

But $(3 \mathrm{~b})$ is plainly false $-\boldsymbol{F}$ is a part of itself, and also a part of countless fusions of itself with other objects.

\section{Theory 4}

It was suggested to me that by (3) Tooley means: ${ }^{13}$

\footnotetext{
${ }^{12}$ I consider the possibility that $F$ is an improper part of $F \& G$ in section 3 below.

${ }^{13}$ by an anonymous referee for Philosophical Studies.
} 
(3c) $F$ is a part of $F \& G$, and $F$ is not a part of any universal that is not a part of $F \& G$

But Theory 4 is unacceptable. Suppose that in addition to the law that all $F$ s are $G$ s we have another law that all $F \& G$ s are $H$ s. The theory we are presently considering has the result that $F \& G$ exists only as a part of $F \& G \& H$, which entails that $F \& G$ is a part of $F \& G \& H$. Surely $F \& G$ is not identical to $F \& G \& H ;{ }^{14}$ hence it is a proper part of $F \& G \& H$, and hence (i): $F \& G \& H$ is not a part of $F \& G$. But by transitivity of the part-whole relation, since $F$ is a part of $F \& G$ which is a part of $F \& G \& H$, (ii): $F$ is a part of $F \& G \& H$. By (i) and (ii), (3c) is false, but (3c) is true on Theory 4 , for we originally stipulated that there was a law that $F$ s are $G$ s.

\section{Theory 5}

Some people have informed me that when they hear "exists only as a part of" they hear an assertion of some kind of ontological dependence. Moreover, Tooley begins his discussion of the speculative theory by defining the technical term 'dependent universal': a universal is dependent in a world iff it exists, at that world, only as a part of other universals. This all suggests the following sort of definition:

$x$ exists only as a part of $y$ iff i) $x$ is a part of $y$, and ii) IF $y$ fails to exist, THEN $x$ also fails to exist

Theory 5, which accepts this definition and regards 'part' as being undefined, does not help us derive (4). We have already argued that $F$ 's being a part of $F \& G$ does not straightforwardly imply that all $F$ s are $G$ s. It makes no difference when we add the information that $F$ fails to exist if $F \& G$ fails to exist.

\section{Theory 6}

Theory 6 keeps the definition from the previous section of 'exists only as a part of' and additionally accepts some definition of the form (P). Here it is time to employ an objection that is, I think, telling against both Theory 5 and Theory 6. Let us ask what sort of conditional is involved in the current

\footnotetext{
${ }^{14}$ If it is claimed that these universals are identical, the arguments of section 3 apply.
} 
definition of 'exists only as a part of'. I wrote only a schematic 'IF...THEN'. It seems that all answers are unacceptable. The conditional cannot be the material conditional, for since ' $x$ is a part of $y$ ' entails that $y$ exists, the definition would simply say that $x$ exists only as a part of $y$ iff $x$ is a part of $y$, and we have already considered this possibility in Theories I and 2. Neither can it be the strict conditional of entailment. Entailments are necessarily true if possibly true (assuming $\mathrm{S}_{5}$ modal logic for broad metaphysical possibility). Hence, for any universals $U$ and $U \& V$ where it is possible for $U$ to exist only as a part of $U \& V$, it will be a necessary that: $U$ exists only as a part of $U \& V$ iff $U$ is a part of $U \& V$. Thus, the current definition of 'exists only as a part' collapses to the earlier definition from Theories I and 2 , at least for all universals that can possibly be lawfully related. Neither can the conditional be that of nomic entailment, for this would be a gross circularity. Finally, the conditional cannot be the counterfactual conditional, again on pain of circularity, for the analysis of such conditionals surely must make appeal to laws. ${ }^{15}$

Tooley's speculative theory says that the law that all $F$ s are $G$ s consists in $\boldsymbol{F}$ existing "only as a part of" $F \& G$. Its goal is to provide an illuminating solution to the inference problem. To achieve this goal, Tooley must show that (3) entails (4), and he must show this without appeal to "radical" principles that destroy the value of the speculative theory as genuine illumination. I have found no plausible interpretation of the speculative theory that gives Tooley these results.

\section{The Enhanced Speculative Theory}

We still seek a way to justify Tooley's inference from (3) to (4). I propose now to explore a different strategy which bases the inference from (3) to (4) on the Indiscernibility of Identicals. I do not pretend here to be interpreting Tooley. But as we have seen, what he does say about the derivation of (4) from (3) is inadequate; moreover the following discussion has intrinsic interest.

Let us suppose that 'exists only as a part' means simply 'is a part of'. Thus, we interpret (3) as (3a). We have a law that $F$ s are $G$ s just when $F$ is a part of $F \& G$. The next step is to argue for the following principle:

\footnotetext{
${ }^{15}$ See, for example, Lewis's discussion in Lewis (1973, 72-77).
} 
(5) For any conjunctive universal $U \& V, U \& V$ is a part of $U$ and also a part of $V$.

(5) indeed represents progress, for it implies that $F \& G$ is a part of $F$; combined with (3) this yields the result that $F \& G$ and $F$ are identical (since each is a part of the other). But if they are identical, then by the Indiscernibility of Identicals anything that instantiates $F$ also instantiates $F \& G$. We have derived (4) from (3a).

But why should we accept (5)? It seems to be another radical principle. One way it might be supported is if the part-whole relation between universals were construed as being analogous to the subclass relation between classes determined by universals. The class of $F \& G$ s is a subclass of the class of $F$ s. Using this as a model, we might offer the following definition:

(DI) Universal $U$ is a part of universal $V=_{\mathrm{df}}$ the class of $U \mathrm{~s}$ is a subclass of the class of $V$ s.

If (DI) defines the part-whole relation among universals, then (5) is indeed true. But (DI) is unacceptable. Suppose it is an accident that all $F$ s are $G$ s. Then all $F$ s are $F \& G$ s, so (DI) says that the universal $F$ is a part of the universal $F \& G$, and hence the speculative theory says that it is a law that $F$ s are Gs. Furthermore, this part-whole relation between universals is reducible to facts about particulars, violating the requirement laid out by Tooley that the necessitation relation must be a relation that holds irreducibly between universals. ${ }^{16}$

We cannot employ (DI) to support (5). Another natural way to understand 'part' here is suggested by the picture of immanent universals painted by Armstrong. ${ }^{17}$ On Armstrong's view, universals are wholly present when they are instantiated; they are literally parts of their instances. Perhaps a little imaginative fiction might stimulate intuitions here. Imagine beginning with a bare particular. Now begin adding universals. Suppose we add $F$, and then add $G$. Since the particular is now an $F$ and a $G$, the universal $F \& G$ is also present now. By adding $F$ and $G$, we thereby added $F \& G$. This suggests the view that $F$ and $G$ are each a part of $F \& G \cdot{ }^{18} F \& G$ was present in the particular when (and only when) we added both $F$ and $G$. If I add two gallons of water to a bucket, each gallon is a part of the total quantity of water that I added.

\footnotetext{
${ }^{16}$ Tooley $(1987,76)$.

${ }^{17}$ See Armstrong (1978a,b).

${ }^{18}$ Armstrong seems to accept this view in Armstrong (1978b, $30 \mathrm{ff}$.)
} 
On this picturesque partial account of the part-whole relation among universals, conjunctive universals have parts: their conjuncts. I find this an intuitive way to think about part-whole relations between universals, but it is of no help for it does not imply (5), the principle we seek. Rather, it implies

$\left(5^{\prime}\right)$ for any conjunctive universal $U \& V, U$ is part of $U \& V$

In fact, $\left(5^{\prime}\right)$ is fatal to the enhanced speculative theory, according to which there is a law that $U$ s are $V$ s iff $U$ is a part of $U \& V$, for $\left(5^{\prime}\right)$ generates a law whenever we have a conjunctive universal.

I do not see any way to argue that (5) is true. But suppose that, contrary to appearances, (5) could be established. Better still, since the point of establishing (5) is that it has the consequence that (3a) implies the identity of $F$ and $F \& G$ (in the world in question), we could circumvent the problem of establishing (5) by taking the speculative theory simply to be this consequence. Thus, let us assume that the speculative theory states or implies that if there is a law that $F$ s are $G$ s at a given world then the universals $\boldsymbol{F}$ and $\boldsymbol{F} \& G$ are identical at that world.

Isn't this too strong? By the standard principle that identicals are necessarily identical, it follows that it is necessary that $F$ and $F \& G$ (if they exist) are identical. But, since every object is a part of itself, it would then be a necessary truth that $F$ (if it exists) is a part of $F \& G$, and hence the original law would be a necessary truth. (Better: it would hold in any possible world in which the universals exist). Laws would become logically necessary.

This conclusion could be avoided by claiming that the identity between $F$ and $F \& G$ is contingent. More carefully, the claim would be that the sentence ' $F$ $=F \& G$ ' expresses a contingent proposition since the term ' $F \& G$ ' is not a rigid designator. When I discuss the universal that is "the conjunction of $\boldsymbol{F}$ and $G$ ", I am discussing a universal that, in fact, has the property of being the conjunction of those universals. That is, it bears the three place relation conjunction to $F$ and $G$. At another possible world some other universal might bear that relation to $F$ and $G$. Hence, the contingent identity here would be no more mysterious than the fact that Manute Bol is, but might not have been, identical to the tallest player in the National Basketball Association.

This move is implausible. If you speak of a relation among universals called 'conjunction', but tell me that $W$ may bear it at one world to $U$ and $V$, but fail to do so at another world, then I say it is not the conjunction relation. All are agreed that it is part of the meaning of 'conjunction' that 
(6) Necessarily, if object $x$ instantiates conjunctive universal $U \& V$, then $x$ instantiates both $U$ and $V$

But surely it is also part of the meaning of 'conjunction' that

(7) Necessarily, if universal $W$ is the conjunction of universals $U$ and $V$ then necessarily: if object $x$ instantiates $W$ then it instantiates both $U$ and $V$.

To deny (7) is to deny an extremely compelling view of the transworld criteria of identity for conjunctive universals. It seems to have the status of the analogous principle:

(8) Necessarily, if set $\phi=\{x, y\}$, then necessarily: $\phi=\{x, y\}$

(8) is a consequence of the idea that the principle of extensionality for sets extends to transworld identity of sets. Granted, (8) is not implied by standard accounts of set theory, for such theories are not "modalized". But (8) is so natural as to seem definitional. Moreover, in the absence of (8) we would seem to be at an utter loss to answer questions of transworld identity for sets. If sets are not individuated across worlds by membership, then how are they thus individuated? Similarly for conjunctive universals: if we deny (7) then it is difficult to envision the principles governing transworld identity for universals.

I have a final objection to the enhanced speculative theory. Suppose that unit negative charge and spin $\mathbf{I} / \mathbf{2}$ are universals; call them " $U$ " and " $V$ " for short. Now consider some world $w$ in which we have a law that $U$ s are $V$ s, and also a law that $V$ s are $U$ s. The enhanced speculative theory implies that, at $w, U=U \& V$ and that $V=U \& V$ and hence that $U=V$. This means that, at $w$, there is no difference between having unit negative charge and having spin $\mathrm{I} / 2$ ! This seems wrong. Surely, even if it were a matter of law that these properties were present in exactly the same objects, we could still distinguish in a given object its feature of having unit negative charge and its feature of having spin $I / 2$.

\section{Conclusion}

Tooley's speculative theory, I have tried to show, is beset with difficulties. I warned that this theory threatens to fail in its goal of illuminating the possible 
nature of a necessitation relation if it employs radical principles about parts and wholes and universals. Secondly, I argued that regardless of how we interpret the crucial phrase 'existing only as a part of', the speculative theory cannot solve the inference problem without appealing to such radical principles. Finally, I considered and rejected a version of Tooley's speculative theory that attempts to solve the inference problem using the Indiscernibility of Identicals.

I conclude that the speculative theory fails. Tooley must fall back to the stipulative theory and live with the lack of an illuminating solution to the inference problem. But this is not to admit defeat. While it would be nice to have a more complete account of its workings, we may view the introduction of the necessitation relation as a theoretical posit, analogous to the positing of subatomic particles in physics to account for various data.

\section{References}

Armstrong, David M. (1978a). Nominalism and Realism, volume I of Universals and Scientific Realism. Cambridge: Cambridge University Press.

- (1978b). A Theory of Universals, volume 2 of Universals and Scientific Realism. Cambridge: Cambridge University Press.

- (1983). What Is A Law Of Nature? Cambridge: Cambridge University Press. Lewis, David (1973). Counterfactuals. Oxford: Blackwell.

- (1986). Philosophical Papers, Volume 2. Oxford: Oxford University Press.

Tooley, Michael (1987). Causation: A Realist Approach. Oxford: Clarendon Press.

van Fraassen, Bas (1989). Laws and Symmetry. Oxford: Clarendon Press. 